\title{
The Eleventh Hour: Neurosyphilis, Still Fashionable but a Controversial Diagnosis
}

\author{
Rodica Bălaşa* \\ University of Medicine and Pharmacy of Tirgu Mures, Romania
}

Syphilis is a consequence of a symbiotic relationship between Treponema pallidum and humankind.

The spirochete is nowadays well characterized in shape and in length, and its entire genome is sequenced. Despite all these, confirmation of infection is based on serologic tests. The diagnosis of nervous system disease heavily depends on examination of the cerebrospinal fluid [1-4].

Neurologic involvement is generally dichotomized into early/secondary (acute meningitis, cranial nerves involvement) or late/tertiary (all the rest of the manifestations) [1].

In the current issue of Journal of Critical Care Medicine, Bologa et al. present the utility of having meningovascular syphilis in mind as a possible diagnosis, in an apparent average case of an 84-year-old patient diagnosed with stroke [5]. The obliterative endarteritis that involves the intracranial arteries causes stroke. Symptomatic involvement of the central nervous system (CNS) is scarce, found in $4-6 \%$ of neglected patients. Among neurosyphilis cases, this type of CNS involvement occurs in approximately a forth of untreated patients $[1,2]$.

The spirochetal infections of the nervous system have two main actors: Treponema Pallidum and Borrelia Burgdorferi sensu lato. There are numerous similarities between these 2 infections, due to biologic characteristics of spirochetes: a wide mythology, both being called the great imitator; after 1950 the types of central nervous system (CNS) involvement were described in a scientific manner; both are chronic; the immune reaction can be demonstrated by the presence of antibodies against major epitopes (a small number of surface antigens are expressed on the spirochete's surface but the expressed epitopes might be changed during the infestation); a close contact with a vector is required for spirochetal spreading, demonstrating the lability of this organism in vitro; the infestation is marked by impressive painless (probably secondary to poor host immune response) cutaneous involvement; a good motility and adherence to cell-surface molecules allows spirochetes to penetrate the blood-brain barrier and disseminate into the CNS; a various but atypical CNS pathology is produced (an approach based on phenomenology instead of pathophysiology) but fortunately only a small number of patients become clinically manifest; a diagnosis of later CNS disease demand advanced techniques for cerebrospinal fluid assay; luckily, both spirochetes are sensitive to the same family of antibiotics $[1,3,6]$.

An interesting discussion is that cranial syphilitic vasculitis differs from other cerebrovascular disease through the site of damage and not the mechanism. Prodromal manifestations are more frequently found in meningovascular syphilis (headaches, mental status change, meningismus) compared with classical stroke cases. The percentages of the symptoms as described in specialized literature are: headache $10 \%$, dizziness $10 \%$. The arterial territory most frequently affected is the middle cerebral artery territory. It is still unknown which factors lead to the development and evolution of this chronic tertiary syphilis. The vascular lesions are due to an endarteritis with perivascular inflammation that obliterates vasa vasorum. The endarteritis consists in an intimal fibroblastic proliferation, decreasing the media thickness, and in the periphery plasma cells and lymphocytes infiltrate the adventitia [1-3].

The old tertiary syphilis classification comprised 3 types: cardiovascular syphilis, neurosyphilis, and late benign also known as gummatous syphilis. Lately, researchers have gathered clinical and laboratory data from neurosyphilis cases and compiled them into 6 different diagnostic types: first category - neuropsychiatric (most frequently found) disorders such as dementia, 
delirium, psychosis; second category - ischemic stroke with an acute onset of a focal neurological deficit; third category, with ocular involvement which amounts to optic nerve dysfunction followed sometimes by visual loss or uveitis; fourth category, where tabes dorsalis is included, with myelopathy of various types of onset i.e. acute, subacute, or chronic spinal cord syndromes; fifth category - the epileptic type, presenting partial seizures or myoclonus; sixth category, involving the brain stem and the cranial nerves. The 84 -year-old patient reported in the article under discussion can be classified in the second category. All the prodromal symptoms of the patient were of CNS origin (dizziness, memory loss, insomnia, headache). In a previous publication from 2014, we reported the case of a 64 year-old patient that presented hints of meningovascular syphilis, syphilitic amyotrophy and general paralysis maybe a seventh category of tertiary syphilis [1-4].

Regarding the risk of neurosyphilis, Caucasians are 2-3 times more predisposed than the rest of populations, and it is more frequent in males than in females. The case presented was a female that, like other referred-to cases, contracted the infection most probably long before the disease expressed clinically, placing this pathology among progressive chronic infections.

Spirochetal infection diagnosis in the tertiary stage is exclusively done by serologic tests. We must not forget that patients infected with spirochetes frequently have elevated titers of anticardiolipin antibodies (a possible explanation would be the putative interaction of the host's lipoproteins with the spirochete). Diagnostic tests (that are very sensitive but lack specificity) from this group of "reaginic" antibodies are: Venereal Disease Research Laboratory (VDRL), rapid plasma reagin, Wassermann test, Hinton tests. A further test in positive patients to these reaginic tests is needed: microhemagglutination for Treponema Pallidum, Fluorescent treponemal antibody. The importance of the VDRL test remains when evaluating the response to a certain antibiotic. An infection of the CNS requires the thorough examination of the CSF: CSF VDRL is reliable (a positive result is perceived as part of the diagnosis). A slight increase of CSF leukocytes was found in the case reported by Bologa et al., similar to the ones found in the specialized literature. From the theoretical point of view, it is still highly debatable whether the antibodies against Treponema pallidum are generated inside the CNS $[3,7,8]$.
Lately, due to the increase of syphilis infection even in the most unlikely patients, screening for spirochetal infection is advisable, although interpreting the tests might be a source of problematic false-positive or falsenegative results $[4,6]$. The diagnosis is equally important since neurosyphilis has a curative treatment which is easy to administer, generally without any adverse events or significant costs [7]. This is also the case of the reported 84-year-old patient.

At first glance, stroke pathology in an 84-year-old woman is a common occurrence, as well as syphilis vascular involvement of the CNS. The importance of case reports resembling the one currently under discussion consists of the different final image compared to each trivial scattered piece. The discussion of the pathophysiology mechanism of CNS involvement by an infection first described in 1530 remains still open in 2017.

\section{DDISCLOSURE}

The author reports no disclosure relevant for this manuscript.

\section{REFERENCES}

1. Halperin JJ: Spirochetal infection of the nervous system. In Aminoff M, Josephson SA (eds): Aminoff's Neurology and General Medicine, Fifth Edition. London: Elsevier. 2014, pp.81731

2. Centers for Disease Control and prevention (CDC) Summary of Notifiable Diseases-United States, 2010. MMWR Morb Mortal Wkly Rep. 2012;59:25

3. Park UI, Chow JM, Bolan G, et al. Screening for syphilis with the treponemal immunoassay: analysis of discordant serology results and implications for clinical management. J Infect Dis. 2011;204:1297-2004

4. Balasa R, Bajko Z, Motataianu A, Maier S. Pitfalls in the diagnosis of neurosyphilis- case report and literature review. Acta Medica Transilvanica. 2014;4:201-3

5. Bologa C, Lionte C, Halit D, Luca C. Neurosyphilis Masquerading as Stroke in an 84-year-old. J Crit Care Med. 2017;3(2):70-72.

6. Mattei PL, Beachkofsky TM, Gilson RT, Wisco OJ. Syphilis: a reemerging infection. Am Fam Physician. 2012;86(5):433-40

7. Ghanem KG, Workowski KA. Management of adult syphilis. Clin Infect Dis. 2011;53(Suppl 3):S110-28

8. Sparling PF. Diagnosis of neurosyphilis: new tools. Sex Transm Dis. 2010;37(5):288-9 